\title{
¿POR QUÉ FINLANDIA PUEDE PRESCINDIR DE ACCOUNTABILITY FORMAL EN EDUCACIÓN Y POLONIA NO? ${ }^{1}$
}

\author{
Francisca Dussaillant y Eugenio Guzmán²
}

\begin{abstract}
RESUMEN
El objetivo de este documento es aplicar la teoría del capital social (cuyo principal indicador es la confianza) al problema específico del accountability en educación. La evidencia indica que países de bajo capital social (como Polonia) y países de alto capital social (como Finlandia) han podido desarrollar políticas educacionales exitosas, según las mediciones internacionales. Uno de los determinantes del éxito polaco en su reforma educacional, que comenzó principios de la década pasada, fue la instauración formal de un sistema de accountability centralizado. Por su parte, Finlandia tiene un sistema basado en la confianza y en el profesionalismo docente, donde las pruebas estandarizadas no tienen cabida. Los países latinoamericanos, entre los que se cuenta Chile, tienen bajos desempeños en las pruebas internacionales. Es natural, por tanto, que miren hacia las experiencias exitosas, con el objetivo de aprender de ellas y eventualmente adoptar alguna de sus políticas. Se argumenta que el sustrato social sobre el que se construye una determinada institucionalidad es fundamental para determinar el éxito o fracaso de esas adaptaciones. Los países latinoamericanos tienen niveles muy bajos de capital social, al igual que Polonia y, por lo tanto, debieran aprender de la reforma educacional polaca. El caso finlandés parece menos aplicable en Latinoamérica, ya que solo países con alto capital social pueden funcionar sobre la base de instituciones de accountability informales.
\end{abstract}

Palabras clave: capital social, confianza, accountability, reforma educacional

\section{WHY CAN FINLAND DO WITHOUT ACCOUNTABILITY IN EDUCATION, BUT POLAND CANNOT?}

ABSTRACT

The objective of this article is to apply the theory of social capital (whose principal indicator is confidence) to the specific problem of accountability in education. The evidence suggests that countries with low social capital (such as Poland) and countries of high social capital (such as Finland) have been able to develop successful educational policies, according to international measures. One of the determinants of the Polish success in education reform, which began at the beginning of the previous decade, was the formal installation of a centralized accountability system. Finland, on the other hand, has a system based on confidence and teacher professionalism, where standardized exams do not have a place. The Latin-American countries, among which is Chile, exhibit low performance on international exams. It is only natural, therefore, to look towards the successful experiences, with the objective to learn from them and eventually adopt some of their policies. It is argued that the social background upon which a determined institution is built is fundamental for determining the success or failure of these adaptations. The Latin-American countries, Chile included, have very low levels of social capital, same as Poland, and thus should try to learn from Poland's educational reform. The case of Finland seems less applicable in Latin-American as only countries with high social capital can function on the basis of informal institutions of accountability.

Keywords: social capital, confidence, accountability, educational reform.

1 Agradecemos a Juan Pablo Couyoumdjian, José de la Cruz Garrido y Massiel Guerra por sus comentarios y sugerencias. Las opiniones aquí vertidas son de carácter individual (no representan a las organizaciones en las que participamos). Cualquier error u omisión es de exclusiva responsabilidad nuestra.

2 Facultad de Gobierno, Universidad del Desarrollo, Santiago, Chile (ambos autores). Contacto: eguzman@udd.cl 


\section{Introducción}

La rendición de cuentas o accountability utilizada como mecanismo para evaluar el desempeño de una escuela a través de la medición de los aprendizajes de sus estudiantes, tiene una prevalencia cada vez mayor en el mundo y es parte de la institucionalidad educacional de muchos países. La mayoría de los países de Europa occidental ${ }^{3}$, Estados Unidos, distintas provincias de Canadá y varios países latinoamericanos, incluyendo Chile, tienen algún sistema de examinación centralizado desde hace más de una década (Figlio $\&$ Loeb, 2008). Australia también se sumó a esta tendencia en el año 2008 al instalar su sistema nacional de medición de lectura y matemáticas denominado NAPLAN (Gable \& Lingard, 2013), y Sudáfrica aplicó por primera vez en 2011, a nivel censal, su medición nacional anual (ANA) (Department of Basic Education, 2011).

En general, existen tres tipos de rendición de cuentas (Anderson, 2005): el primero de ellos tiene que ver con el cumplimiento de las normas existentes (regulaciones), el segundo con adhesión a los estándares profesionales (currículo) y el tercero, con los resultados (aprendizaje y desempeño). Una acepción común de rendición de cuentas o accountability en educación, que será la que utilizaremos en este documento, se relaciona con el tercero de estos tipos y supone el uso de información o datos a nivel centralizado para mejorar el desempeño de los estudiantes. La mayor parte de los sistemas nacionales de rendición de cuentas genera incentivos, en la forma de sanciones o premios (implícitos o explícitos), a las escuelas sobre la base de los resultados de sus estudiantes en pruebas estandarizadas. Estos incentivos pueden operar a través de la acción gubernamental directa, o bien, a través de la provisión de información (Figlio \& Loeb, 2008).

El surgimiento de la rendición de cuentas o accountability como política educacional está íntimamente relacionado con el movimiento de las reformas basadas en estándares. El objetivo de estas reformas es identificar un conjunto de estándares claros, objetivos y medibles, ambiciosos pero alcanzables, para las áreas fundamentales del aprendizaje. El cumplimiento de estos se mide a través de pruebas

3 Ver, por ejemplo, EACEA, 2009.. 
estandarizadas de manera de identificar a aquellos que se van quedando atrás y estimular la mejora en las escuelas.

Las políticas de accountability surgen como respuesta al problema de agencia. Se habla de situación o contrato de agencia cuando una persona (principal) encarga a otra (agente) la gestión de una actividad, dándole autonomía para la toma de decisiones, mientras que el agente por su parte se compromete a defender los intereses del principal (Jensen \& Meckling, 1976). La relación de agencia implica la existencia de un riesgo moral, es decir, la posibilidad de que el agente trabaje en pos de sus intereses personales en perjuicio de los intereses del principal. Estos comportamientos oportunistas solo pueden darse en un contexto de información asimétrica: el principal no conoce el detalle de las acciones del agente. Cuando aparecen los mecanismos de monitoreo, la información pasa a ser simétrica y el problema del riesgo moral se desvanece, ya que el agente está siendo observado. En el caso de la política educacional, el monitoreo realizado a través de políticas de rendición de cuentas solucionaría, al menos en parte, las asimetrías de información que existen entre los agentes (profesores, sostenedores) y el principal (gobierno, familias) ${ }^{4}$.

En general, la evidencia indica que las políticas de rendición de cuentas han movilizado a los sistemas educacionales (Figlio $\&$ Loeb, 2011), especialmente cuando han estado atados a incentivos explícitos, aunque también cuando solamente funciona el componente informacional, que es un movilizador implícito. La sola información respecto del desempeño de un establecimiento puede movilizar a dichos sistemas, ya que se asocian a esta consecuencias respecto de su reputación, de presión para mejorar por parte de padres y comunidad escolar, o de cambios en la demanda de matrícula.

Los sistemas de rendición de cuentas, entonces, tienen el beneficio potencial de alinear el esfuerzo de los profesores y sostenedores con la consecución de los objetivos de los stakeholders

4 La respuesta a si, en el contexto de las políticas educacionales, el "principal" es el gobierno o las familias, es fuente de discusión. Para los efectos de este análisis, esa discusión es irrelevante en la medida en que supongamos que los objetivos de ambos actores están perfectamente alineados. 
(familias, gobiernos, etc.), en lo que se refiere a los aprendizajes. Sin embargo, están limitados por el hecho de que algunos de los aspectos valorados por estos son difíciles de medir (por ejemplo, áreas de ética laboral, ciudadanía, pensamiento crítico). Otro efecto no deseado es el teaching to the test, que ocurre cuando el profesor se centra en enseñar solamente para que los alumnos aprueben el examen, o también el comportamiento estratégico, que sucede cuando la escuela se enfoca en estrategias para mejorar artificialmente los puntajes (como etiquetar a los alumnos más desaventajados como con "necesidades educativas especiales", lo que en muchos sistemas los eximiría del examen), antes que en la enseñanza de los contenidos (Figlio \& Loeb, 2011). Todo lo anterior es manejable en gran medida a través de las estrategias de diseño del test (tipo de preguntas, definición de destrezas a medir, entre otros), el diseño institucional acertado de condiciones para rendirlo y la adecuada fiscalización. Esto es un desafío constante de todos los sistemas de medición estandarizada.

\section{Pruebas estandarizadas versus confianza}

Aun cuando la rendición de cuentas a través de examinaciones estandarizadas es una política en auge a nivel mundial, existen algunas naciones que no han implementado estos sistemas y aun así son exitosas. Ejemplo de esto es Finlandia, país que ha mostrado un desempeño ejemplar en pruebas internacionales como PISA (ver Tabla 1). Los finlandeses tienen un sistema educacional exitoso, construido principalmente sobre la base de la colaboración, el profesionalismo docente, la enseñanza personalizada y la responsabilidad basada en la confianza y la equidad. En Finlandia la medición centralizada no existe, porque la sola idea de una política de este tipo va en contra del espíritu de su institucionalidad educacional (Sahlberg, 2011).

Otras naciones, sin embargo, han logrado el éxito por una vía distinta a la finlandesa. En estas la implementación de pruebas estandarizadas ha sido determinante en su mejora educacional, un ejemplo es Polonia, que en el año 1999 aproximadamente comenzó un proceso de reforma educacional que lo ha transformado en el país con mayores avances en la prueba PISA. Polonia logró mejoras en sus resultados de matemática a una tasa anual de 2.6 puntos PISA, lo que 
llevó al país desde un puesto por debajo del promedio OCDE en 2003 a resultados similares a los de los finlandeses en 2012 (ver Tabla 1). Los resultados exitosos fueron evidentes en toda la distribución de puntajes: las mejoras se observaron tanto en el grupo de estudiantes polacos con puntajes deficientes, como en aquellos con desempeños destacados, y se replicaron también en lenguaje y ciencias. Un ingrediente importante de la reforma llevada a cabo por este país fue la introducción de un sistema centralizado de pruebas estandarizadas y exámenes. Las pruebas estandarizadas se realizan al final de la primaria (sexto año) con fines informacionales y de comparabilidad. Por otro lado, un examen que los estudiantes deben rendir a finales de la secundaria inferior (tercer año) tiene consecuencias directas para estos, ya que define su situación en el proceso de selección para la secundaria superior (de cuarto a sexto año). A fines de sexto año otro examen con consecuencias individuales para los alumnos define su admisión en la educación terciaria (OCDE, 2013).

\section{Ejemplos para Chile y Latinoamérica}

Tanto Polonia como Finlandia podrían ser catalogados hoy como "ejemplos a seguir" por Chile, país que tal y como se observa en la Tabla 1, está muy por detrás de ellos en lo que se refiere a los resultados de aprendizaje de sus estudiantes. Aun cuando Chile destaca entre sus pares ${ }^{5}$, se encuentra muy lejos del promedio OCDE y más lejos aún de los países que se utilizan aquí como ejemplo. Así, en Chile la mitad de los estudiantes tiene puntajes en la prueba que están por debajo del nivel 2, que representa el estándar mínimo de alfabetización matemática. De acuerdo con PISA solo quienes logran el nivel 2 o superior están preparados para participar en la sociedad moderna (OCDE, 2014): Finlandia y Polonia ubican a más del $85 \%$ de sus estudiantes por sobre el estándar mínimo y superan por órdenes de magnitud a Chile y sl resto de los latinoamericanos, si comparamos su capacidad de colocar estudiantes en los niveles de desempeño superiores ( 5 y 6 ).

5 En la prueba PISA 2012 Chile apareció con resultados superiores a los de cualquier país latinoamericano participante (Argentina, Brasil, Colombia, Costa Rica, México, Perú y Uruguay). 
¿Qué hubiera pasado si Polonia optaba por copiar el sistema finlandés, en términos de su enfoque de accountability, en vez de desarrollar su reforma tal cual lo hizo? ¿Cuál es el camino que debiese seguir un país latinoamericano como Chile, si tuviera que salir a "copiar" experiencias exitosas en el ámbito educativo, específicamente en lo que se refiere a la rendición de cuentas?

Este tipo de preguntas acerca de los modelos a seguir son difíciles de contestar, ya que los análisis se pueden hacer en múltiples dimensiones y a través de diferentes enfoques. Sin embargo, en el caso particular de las políticas de accountability, una dimensión muy manifiesta tiene que ver con el sustrato social sobre el que se construye la política, donde el nivel de confianza imperante en el entorno es un ingrediente clave. Nuestro argumento es que Polonia se parece más a Chile (y a Latinoamérica) que Finlandia en aspectos sociales clave que son determinantes para el éxito o el fracaso de una política.

Tabla 1: Resultados en la prueba PISA 2012

\begin{tabular}{l|c|c|c|c|c}
\hline & x Matemática & $\begin{array}{c}\text { \% Bajo nivel 2 } \\
\text { en matemática }\end{array}$ & $\begin{array}{c}\text { \% nivel 5 0 6 } \\
\text { en matemática }\end{array}$ & x Lenguaje & x Ciencia \\
\hline Chile & 423 & 51.5 & 1.6 & 441 & 445 \\
\hline Finlandia & -519 & 12.3 & 15.3 & -524 & -545 \\
\hline Polonia & 518 & 14.4 & 16.7 & 518 & 526 \\
\hline
\end{tabular}

Fuente: OCDE (2014).

A continuación desarrollaremos en profundidad los argumentos que nos llevan a pensar que la política de rendición de cuentas finlandesa no hubiera tenido éxito en Polonia. El análisis nos permitirá concluir que los polacos, al elegir un sistema de monitoreo centralizado, habrían hecho lo correcto, y en eso radicaría al menos parte del éxito de su reforma. Por último, la idiosincrasia latinoamericana -incluida la chilena-, se asemeja más a la polaca que a la finlandesa, por lo que lo que si queremos emular algún modelo de reforma exitoso debiésemos mirar hacia Polonia. 


\section{Motivación intrínseca versus motivación extrínseca}

El problema de agencia definido en la introducción describe el comportamiento de un agente racional, que busca maximizar su bienestar, lo que a veces entra en conflicto con la maximización del bienestar del principal. La evidencia empírica indica que este modelo es aplicable en una gran cantidad de escenarios. Sin embargo, los seres humanos no nos movemos solamente por consideraciones racionales, sino que también somos sujetos, por ejemplo, de emociones (Damasio, 2003). Esto ha sido comprobado en numerosas ocasiones en la literatura sicológica y en la economía del comportamiento. Varios experimentos han demostrado la existencia de una serie de anomalías en el comportamiento, resultados raros en la medida en que contradicen lo que se esperaría observar en el comportamiento de seres solamente racionales (ver, por ejemplo, Kahneman, Knetsch, $\&$ Thaler, 1986).

Una de estas anomalías tiene que ver con el eventual desplazamiento de la motivación intrínseca por parte de motivadores extrínsecos (como el dinero). Cuando una tarea se realiza sobre la base de una motivación intrínseca, el individuo aparentemente no recibe recompensa más allá de su satisfacción por realizar la actividad propiamente tal. Por otro lado, cuando hay un pago involucrado, la motivación para hacer la tarea es externa al individuo (extrínseca). Pues bien, se ha demostrado que cuando en una actividad normalmente realizada sobre la base de motivaciones intrínsecas (por ejemplo voluntariado) aparece una motivación extrínseca (pago), estas no se suman sino que la motivación extrínseca desplaza a la intrínseca, que se extingue. Como a veces la motivación intrínseca es más fuerte que la extrínseca, entonces agregar estos últimos motivos podría disminuir (y no aumentar) el esfuerzo que el agente realice para hacer un buen trabajo (Frey, 2008). Este costo oculto de los premios (Lepper \& Greene, 1978) es evidente en entornos sociales donde la motivación intrínseca es alta. Así, existen ciertos ambientes en los que el accountability, que es un incentivo extrínseco, podría redundar en una disminución de, por ejemplo, el esfuerzo realizado por los docentes en pos de los aprendizajes. 
Lo que es clave es que estos efectos no deseados de los sistemas de accountability serían relevantes solamente en la medida en que los individuos tuviesen, en primer lugar, las suficientes motivaciones intrínsecas para esforzarse en hacer bien su trabajo. Ello requiere que las actividades propiamente tales sean entretenidas e interesantes, cosa que no ocurre siempre (Ryan \& Deci, 2000), o que los individuos funcionen en una sociedad con un alto nivel de valores prosociales. Estos valores prosociales intrínsecos son los que motivan a realizar un trabajo bien hecho. Quienes poseen esos valores son individuos confiables, que no requieren de un control externo. Autores tan relevantes como la premio nobel de economía Elinor Ostrom utilizan el término confiabilidad (trustworthiness, en la cita que sigue) para denominar el resultado de la existencia de valores y motivaciones prosociales.

"We emphasize that individuals' intrinsic values are an independent reason for behaving cooperatively and reserve the term trustworthiness primarily to refer to such nonselfish motives" (Ostrom \& Ahn, 2009, p. 25-26). ${ }^{6}$

Es posible que en una sociedad de individuos con un nivel alto de valores prosociales, es decir, una sociedad de individuos confiables, la rendición de cuentas basada en pruebas estandarizadas tenga como efecto desplazar la motivación intrínseca. Esto a la larga no solo erosiona su capital social sino que, si suponemos que el nivel de motivación intrínseca original es muy alto, puede redundar en una disminución de la calidad de la educación. En otras palabras, podría argumentarse que en un ambiente donde los profesores tienen alta motivación intrínseca por la enseñanza efectiva, la introducción de mecanismos externos como las pruebas estandarizadas podría ser perjudicial para la calidad de la educación. Esto ocurriría si la contribución del mecanismo extrínseco (prueba estandarizada) es menor que la del mecanismo intrínseco que se pierde. Como veremos más adelante, Finlandia parece ser una de estas sociedades. En ambientes de baja motivación intrínseca, asociada con niveles bajos de valores prosociales (capital social), debiese ocurrir lo contrario.

6 Hacemos hincapié en que los valores intrínsecos son una razón independiente para el comportamiento cooperativo y nos reservamos el término confiabilidad principalmente para referirnos a tales motivos no egoístas 


\section{La relación entre confianza y confiabilidad}

Gambetta (2000) define confianza como la probabilidad (o creencia subjetiva) de que un agente asigne a otro (individuo o grupo de agentes) una determinada acción. Entonces, el agente que confía toma en cuenta, en sus decisiones de acción, el riesgo de que el agente en el que se está confiando no actúe de manera recíproca (Ostrom $\&$ Walker, 2003). En teoría, la creencia subjetiva de que el otro es confiable podría ser independiente de las condiciones objetivas. Es posible que un individuo confíe en otro aun cuando este último no actúe de manera confiable. Sin embargo, es razonable suponer que la confianza no es sostenible en el tiempo si el comportamiento del individuo en el que esta se deposita esta no se ajusta a lo esperado (Yamagishi, Kikushi \& Kosugi, 1999; Yamagishi, 2001). En otras palabras, es difícil encontrar confianza donde no hay confiabilidad.

Según Ostrom y Walker (2003), la confiabilidad es una característica interna de los individuos que no siempre puede ser explicada como respuesta racional a algún sistema de incentivos. Así, los autores postulan que la confiabilidad es una preferencia, una razón independiente que empuja al individuo hacia la cooperación. La confianza generalizada, es decir, la confianza en otro al que no se conoce, pasa a ser una expectativa que refleja el nivel promedio de confiabilidad de una sociedad. Este nivel promedio de confianza no es el mismo en diferentes comunidades o naciones, lo que redunda en que en distintos lugares los problemas de acción colectiva son resueltos de maneras diferentes.

La confianza generalizada es rutinariamente medida en muchas sociedades a través de encuestas. La fórmula más común para medirla la encontramos en el formato empleado en el World Values Survey (WVS), un proyecto de investigación global cuyo objetivo es explorar los valores y creencias de las personas, cómo estas cambian en el tiempo y cuál es el impacto social y político que tienen. Desde 1981 se han realizado encuestas representativas en casi 100 países, en algunos de ellos más de una vez, permitiendo así la comparabilidad intertemporal. La encuesta utilizada por WVS incluye la siguiente pregunta, que se utiliza para medir los niveles de confianza general en las poblaciones: 
"Hablando en general, ¿diría usted que se puede confiar en la mayoría de las personas o que uno nunca es lo suficientemente cuidadoso en el trato con los demás?". El entrevistado debe elegir una de las dos alternativas. A partir de los datos de WVS distintos autores muestran que existe una alta consistencia y estabilidad de las respuestas en el tiempo, lo que implica que el indicador de confianza que surge de esta encuesta posee la suficiente validez y confiabilidad (Delhey \& Newton, 2003).

En la línea de la discusión teórica previa, que anticipa una estrecha relación entre confianza generalizada y confiabilidad promedio de las personas en una sociedad, Glaeser, Laibson y Sacerdote (2000), a través de una serie de experimentos concluyen que la respuesta a la pregunta de confianza del WVS, más que revelar cuán confiada es una persona, refleja cuán confiable es ella. En la misma línea, Vyrastekova \& Garikipati (2005) reportan que en sus experimentos, los individuos confiables tendían a ser también los que reportaban confiar más en los demás. Resultados similares fueron obtenidos por Bellemare y Kroeger (2007) y por Sapienza, Toldra y Zingales (2007).

La Tabla 2 muestra el índice de confianza generalizada en Finlandia, Polonia y Chile. Podemos ver allí que en Finlandia los niveles de confianza interpersonal son muy altos. El 58\% de los encuestados de ese país indica que "se puede confiar en todas las personas". Por otro lado, tanto Chile como Polonia muestran niveles de confianza mucho menores ( $12,4 \%$ y $18,1 \%$ respectivamente), y se parecen bastante entre ellos. En efecto, en un análisis similar (sobre la base de otras encuestas) cuando se estudia el grupo completo de países pertenecientes a la OCDE, las sociedades de los países nórdicos son las que reportan mayores niveles de confianza, con Dinamarca, Noruega, Finlandia y Suecia encabezando el ranking y por encima del promedio. Por su parte, Polonia se ubica en el puesto número 22 (por debajo del promedio OCDE) y Chile en el último lugar (30), por debajo incluso de países como México y Turquía (OCDE, 2011).

La WVS contiene también una pregunta que podría asociarse directamente a la percepción del entrevistado respecto de la prevalencia de virtudes prosociales en su comunidad. La pregunta 
"¿Usted cree que la mayoría de las personas se aprovechará de usted si tiene la posibilidad, o actuará limpiamente?" pide al entrevistado que responda utilizando una escala de 1 a 10, donde 1 es "La gente intentará aprovecharse" y 10 es "la gente actuará limpiamente". Los resultados, presentados en la Tabla 3, nuevamente evidencian la similitud entre Chile y Polonia, ambos países en los que pareciera que las actitudes prosociales son más escasas que en la sociedad finlandesa. En efecto, mientras en Chile y Polonia prácticamente uno de cada tres entrevistados declara que, habiendo posibilidad, la gente intentará aprovecharse (responden entre 1 y 3 en la escala), en Finlandia menos de uno de cada diez entrevistados tiene la misma percepción. De la misma manera, mientras en Chile y Polonia la proporción que reporta creer que la gente actuará limpiamente (reporta entre 8 y 10 en la escala) ronda el 15\%, en Finlandia alcanza un 40\%.

Tabla 2: WVS, 2005-2009 (wave 5). Se puede confiar en la mayoría de las personas $(\%)$

\begin{tabular}{l|c|c|c}
\hline & Chile & Finlandia & Polonia \\
\hline Se puede confiar en la mayoría de las personas & 12,4 & 58 & 18,1 \\
\hline Unonunca es lo suficientemente cuidadoso en el trato con los demás & 86,1 & 40,5 & 77,3 \\
\hline No responde & 0,8 & 0 & 0,2 \\
\hline No sabe & 0,7 & 1,5 & 4,4 \\
\hline $\mathrm{N}$ & 1.000 & 1.014 & 1.000 \\
\hline
\end{tabular}

Fuente: World Values Survey Wave 5 (2005-2008).

Tabla 3: WVS, 2005-2009 (wave 5). ¿Usted cree que la mayoría de las personas se aprovechará de usted si tiene la posibilidad, o actuará limpiamente?

\begin{tabular}{l|c|c|c}
\hline $\begin{array}{l}\text { Escala } 1 \text { a } 10 \text { con } 1=\text { "La gente intentará aprovecharse" y } 10= \\
\text { "La gente actuará limpiamente" }(\%)\end{array}$ & Chile & Finlandia & Polonia \\
\hline 1 a 3 & 31,6 & 8,3 & 29,9 \\
\hline 4 a 7 & 47,5 & 51,8 & 45,8 \\
\hline 8 a 10 & 17,5 & 39,5 & 13,6 \\
\hline No sabe/no responde & 3,4 & 0,4 & 10,7 \\
\hline N & 1.000 & 1.014 & 1.000 \\
\hline
\end{tabular}

Fuente: World Values Survey Wave 5 (2005-2008). 


\section{Confianza y capital social}

Los índices de confianza han sido interpretados en la literatura de una manera más amplia que la simple constatación de cuán confiados y confiables son los individuos de una determinada sociedad. De hecho, la confianza es considerada por muchos autores como uno de los mejores (sino el mejor) proxy para medir un concepto bastante más amplio: el de capital social (Coleman, 1990; Putnam, 1993; Brehm \& Rahn, 1997; Narayan \& Pritchett, 1999; Krishna \& Uphoff, 1999; Putnam, 2000; Paldam, 2000; Uslaner, 2001; Zak \& Knack, 2001; Beugelsdijk, de Groot \& van Schaik, 2002; Bjørnskov, 2003; Westlund \& Adam, 2010).

La evidencia de la Tabla 2 indicaría, por lo tanto, que la sociedad chilena es más parecida, en términos de confianza, a la polaca que a la finlandesa y por lo tanto también debiera parecerse en términos de capital social. Esto se ve parcialmente confirmado con los datos de la Tabla 3, que mencionan directamente la existencia de valores prosociales ("la gente actuará limpiamente") en el entorno del entrevistado. La evidencia de esta tabla refuerza la idea de que estos indicadores apuntan a un concepto amplio de capital social, el cual sería significativamente superior en Finlandia que en Polonia o Chile ${ }^{7}$. A continuación nos referiremos más en profundidad a ese concepto.

\section{1. ¿Qué es el capital social y cómo se mide?}

La literatura tanto empírica como teórica plantea problemas a la hora de proveer una sola definición de capital social ${ }^{8}$. Sin embargo, existe bastante consenso respecto de que el término apunta a los fenómenos de asociatividad y conectividad entre individuos. Estos se basan en relaciones de confianza y cooperación que surgen de la interacción y el cumplimiento de normas de reciprocidad. Así, a pesar de la gran

7 Datos de WVS no reportados en este documento muestran que la falta de confianza y de valores prosociales en Chile es un fenómeno que se replica en prácticamente todos los países de Latinoamérica. Por ello, los argumentos que se emiten acá pueden ser extrapolados a toda la región.

8 Según Putnam (2000), en un artículo de Hanifan (1916) fue la primera vez que se utilizó el término capital social. Sin embargo, el concepto había sido desarrollado con bastante anterioridad en la literatura. 
variedad de definiciones el capital social, este puede entenderse como la habilidad de las personas para trabajar mancomunadamente, con el propósito de alcanzar objetivos grupales comunes (Fukuyama, 1995). En otras palabras, el capital social se refiere a la existencia de cierto conjunto de normas (informales), compartidas por un grupo, que permiten la cooperación (Fukuyama, 1997). En definitiva, se trata de normas y redes que facilitan la acción colectiva (Woolcock, 1998). La efectividad de un grupo en el logro de determinadas metas comunes depende finalmente de sus características organizacionales tales como el tipo de redes, normas y confianza social que facilitan la coordinación y cooperación (Coleman, 1988, 1990; Putnam, 1995).

El nivel de cumplimiento de normas, es decir, el grado de fiabilidad o confiabilidad de los integrantes del grupo, genera condiciones de certeza respecto de lo que hacen otros, lo que incentiva la confianza interpersonal y la respuesta recíproca en el cumplimiento de normas sociales informales. Un riesgo permanente de toda red es el aprovechamiento que pueda hacerse de ella (free-riders). Por ello, para medir el capital social se utilizan indicadores como los niveles de asociatividad (participación en organizaciones voluntarias), el tipo de normas compartidas y, finalmente, los niveles de confianza interpersonal y el grado de fiabilidad en el cumplimiento de normas.

En suma, el capital social puede ser definido como el conjunto de recursos individuales (Bourdieu, 1986) y colectivos (Coleman, 1990; Putnam, 2000) necesarios para la acción colectiva. Luego, en la medida en que en una comunidad los individuos posean redes, tengan niveles de confianza altos con quienes interactúan, tengan niveles de cumplimiento de normas de reciprocidad altas y participen en asociaciones y grupos sociales de ayuda mutua, ella estará en condiciones de abordar problemas de acción colectiva más efectivamente que otra comunidad que carezca de alguno de estos atributos.

Respecto de la medición del capital social, Putnam (1993) promueve el uso del número y densidad de redes (vía indicadores de participación en asociaciones) como medida de capital social. No obstante, existe una extensa literatura que ha puesto de relieve una serie de inconvenientes de estos indicadores (ver por ejemplo, Stolle, 
1998 y Beugelsdijk \& Van Schaik, 2005; Lowndes, 2000). Un ejemplo es la dificultad en la comparación entre regiones, países y culturas. Por ejemplo, la participación en organizaciones deportivas tiene significados distintos en diferentes sociedades. Otras críticas al uso de la participación en asociaciones como indicador de capital social es que las actividades desarrolladas por las personas en organizaciones y asociaciones representan solo una pequeña porción de la interacción humana y, en consecuencia, solo una pequeña fracción del proceso de construcción y emergencia de normas surge a partir de la participación en estas (Westlund \& Adam, 2010).

En contraste con el uso de medidas de asociatividad (membrecía), y tal como se mencionó más arriba, la literatura empírica se ha concentrado fuertemente en el uso de la variable confianza (Westlund \& Adam, 2010) como indicador de capital social. Ella resulta ser un atributo simple de manejar y, prima facie, simple de medir (Delhey \& Newton, 2003).

\subsection{Capital social e instituciones}

Un aspecto central en el desarrollo de cualquier sociedad es la calidad de sus instituciones. Estas son los vehículos a través de los cuales las sociedades organizan y coordinan las conductas de sus miembros (North, 1990). De esta manera existirán instituciones más o menos exitosas dependiendo de los efectos que en materia de bienestar, al menos material, generen para sus miembros.

Ahora bien, las instituciones no operan de forma independiente a las conductas y creencias de las personas que pretenden ordenar y coordinar, puesto que su seguimiento depende de cuán internalizadas y aceptadas estén por los individuos. En tal sentido, existe una asociación estrecha entre capital social e instituciones, en lo que respecta por ejemplo a la reducción de costos de transacción (Cersosimo \& Nistico, 2008). Esta asociación se expresa de dos formas: la primera es que el capital social es una fuente para la emergencia de instituciones, y la segunda que un nivel dado de capital social puede operar como un substituto de las instituciones (Ostrom \& Ahn, 2010). 
Las instituciones (formales e informales) exigen de parte de los individuos cierto compromiso, es decir, cumplimiento. En la medida en que los individuos no cumplan los preceptos que se siguen de instituciones formales o informales, estas caerán en desuso.

Si el elemento central en toda institución es la confianza en ellas, es decir, la creencia común del cumplimiento de normas y reglas o de las sanciones por su no cumplimiento, cabe preguntarse, entonces, si en ausencia de instituciones formales es posible el funcionamiento de una sociedad. La respuesta es que sí. En efecto, ciertas falencias institucionales pueden ser suplidas por altos niveles de capital social (Ahlerup, Olsson \& Yanagizawa, 2009). Esto es, probablemente, lo que ocurre en el sistema educacional finlandés, donde pareciera haberse instaurado la cultura de la calidad y el profesionalismo como una institución informal. La confianza es un requisito, una condición para la existencia de una práctica o institución informal.

Por otro lado, debiera esperarse que el impacto marginal de capital social disminuyera con la calidad de las instituciones formales. Ahlerup, Olsson y Yanagizawa (2009) muestran esto para el crecimiento económico de los países. Así, el nivel de capital social de un país se correlaciona con su crecimiento en la medida en que se trate de naciones con institucionalidad formal de baja calidad. Pero esta asociación desaparece cuando se compara países con institucionalidad formal fuerte. Esto es una buena noticia para las sociedades con bajos niveles de capital social: sus debilidades en este aspecto podrán ser contrarrestadas con la emergencia de instituciones formales fuertes. Un ejemplo de este tipo de institución en educación es la rendición de cuentas a través de pruebas estandarizadas centralizadas. Es posible entonces que la formalización de la rendición de cuentas en educación en Polonia haya sido un ingrediente clave de su mejora en la prueba internacional PISA. Asimismo, la baja calidad de los resultados en Chile se podría revertir mejorando la calidad de su sistema centralizado de rendición de cuentas, institución que pudiera no tener una calidad óptima. 


\subsection{Capital social y accountability}

Un aspecto central para el funcionamiento institucional, como ya se mencionó, tiene que ver con el asentimiento de los individuos a las reglas y normas que derivan de ellas. Dicho de otro modo, el funcionamiento de las instituciones depende del nivel de confianza en el cumplimiento de normas por parte de otros individuos y de las sanciones formales e informales que se apliquen a quienes no las cumplan. Es decir, el control de los free-riders resulta crucial. No obstante, esto depende del grado de compromiso de los individuos y la aplicabilidad de sanciones o enforcement formal e informal: si este es precario o débil su efecto sobre el cumplimiento posterior por parte de los free-riders y el resto de una población o comunidad se debilita. En buenas cuentas, en sociedades con bajo capital social, cuando no hay enforcement este se debilita y por ende también lo hace el entorno institucional, ya que las personas dejan de confiar en él.

Aplicando lo anterior al caso particular de la educación, la falta de control formal o informal de los free-riders (que podrían ser profesores o sostenedores cuyo interés principal no esté alineado con la provisión de un ambiente educativo adecuado para sus alumnos) podría conducir a una pérdida importante de confianza en el sistema educacional completo y a un deterioro significativo de las confianzas y el capital social.

En las sociedades con alto capital social el control informal por parte de la ciudadanía es suficiente. Por ejemplo, Boix y Posner (1998) muestran que el capital social influye positivamente en el desempeño gubernamental, en especial porque hace que los ciudadanos vigilen al gobierno más de cerca. Nannicini et al. (2010) aportan evidencia empírica que muestra que el "castigo" electoral al mal comportamiento político es considerablemente mayor en los distritos electorales con alto capital social. En los sectores con bajo capital social, por otro lado, estos controles informales dejan de ser evidentes.

Dicho en otros términos, si los votantes comparten los altos estándares de conducta prosocial que esperan de parte de sus representantes, estarán más dispuestos a informarse y a basar su voto en criterios de bienestar social en lugar de un interés personal más 
miope. Lo mismo debiese ocurrir en contextos educacionales: cuando el capital social es alto, la comunidad debiera tender a castigar el mal comportamiento a través de mecanismos de accountability informal (en la medida que dispongan de alguna información para realizar dicha evaluación). Cuando el capital social es bajo, por otro lado, el control informal se diluye y el accountability formal pasa a tener un rol esencial.

Es importante destacar que la rendición de cuentas es un mecanismo importante para la calidad institucional en todos los entornos. Lo que difiere en los distintos países y sociedades es el tipo de accountability en acción. Cuando el entorno es de alto capital social los mecanismos informales pueden ser los más eficientes. Cuando el capital social es bajo, los mecanismos deben tener un mayor grado de formalidad. Entonces, ipuede subsistir un determinado sistema institucional (política pública) en ausencia de, al menos, algún grado de accountability informal? ¿Puede ser estable una política en un ambiente de bajo capital social y bajo accountability formal? La respuesta a ambas preguntas, a la luz de los antecedentes aquí presentados, pareciera ser que no.

\section{Discusión y conclusiones}

La evidencia indica que países de bajo capital social (como Polonia) y países de alto capital social (como Finlandia) han podido desarrollar políticas educacionales exitosas, según las mediciones internacionales. Uno de los determinantes del éxito polaco en su reforma educacional, que comenzó a principios del año 2000, fue la instauración de un sistema de accountability centralizado. Por su parte, Finlandia tiene un sistema basado en la confianza y en el profesionalismo docente, donde el accountability centralizado y formal no tiene cabida.

Los países latinoamericanos, entre los que se cuenta Chile, tienen bajos desempeños en las pruebas internacionales. Es natural que países con bajos resultados miren hacia las experiencias exitosas, con el objetivo de aprender de ellas y, eventualmente, adoptar alguna de sus políticas. Sin embargo, al momento de elegir los modelos a seguir, es importante tener en cuenta el sustrato social sobre el que se construyó una determinada institucionalidad. Ese tipo de consideraciones son 
fundamentales para anticipar el éxito o fracaso de cualquier adaptación de política. Los países con bajo capital social suplen sus déficits mediante la instauración de instituciones fuertes y de calidad. Solo los países con capital social elevado son capaces de funcionar en ambientes carentes o con muy pocas instituciones formales. Lo anterior ocurre porque donde hay capital social pueden sobrevivir las instituciones informales. En ambientes de bajo capital social, las instituciones informales se desmoronan debido a la acción de los free-riders.

Los países latinoamericanos, Chile incluido, tienen niveles muy bajos de confianza y confiabilidad (proxies de capital social), por lo que su progreso está estrechamente ligado a la calidad y fortaleza de sus instituciones formales. Polonia, un país que también tiene niveles bajos de confianza, ha tenido logros educacionales importantes que lo ubican hoy entre los países con mejores desempeños en la prueba PISA. Estos logros fueron construidos sobre la base de una institucionalidad en la que el accountability formal tiene un rol preponderante. Considerando la relativa similitud entre el sustrato social polaco y el latinoamericano, la reforma educacional polaca puede ser una experiencia que vale la pena mirar en detalle. El caso finlandés no parecería ser aplicable en Latinoamérica, ya que solo países con alto capital social como ese, pueden funcionar en ausencia de institucionalidad o sobre la base de instituciones informales.

Esto no obsta a que la construcción de capital social sea un objetivo relevante y urgente en Latinoamérica, dado que este no solo tiene efectos en la educación, sino en todo el orden institucional, siendo un factor facilitador del bienestar. Las ventajas de vivir en una sociedad confiable y con actitudes prosociales son muchas y, por lo tanto, el camino de la construcción de capital social es un desafío que se debe tomar. Sin embargo, el proceso de generación de capital social en un país es lento y difícil. El proceso de formación de confianza es un fenómeno colectivo y emergente que supone normas, expectativas y el cumplimiento de ambas. La construcción de capital social requiere de tiempo (Ostrom, 2000) o de situaciones sociales anómalas y extremas (desastres, guerras, etc.) (Dussaillant \& Guzmán, 2014) y se destruye, erosiona o diluye con facilidad. Iniciativas educacionales que no entiendan esto -e insistan en suponer que el capital social 
surgirá simplemente "confiando"- podrían conducir a retrocesos aún mayores en las confianzas y a caídas en la calidad de la educación como consecuencia del fracaso de dichas políticas.

\section{Referencias}

Ahlerup, P., Olsson, O, \& Yanagizawa, D. (2009). Social capital vs institutions in the growth process. European Journal of Political Economy, 25, 1-14.

Anderson, J. A. (2005). Accountability in education. UNESCO Educational Policy Series

Bellemare, C., \& Kroger, S. (2007). On representative social capital. European Economic Review, Elsevier, 1(51), 183-202.

Bjørnskov, C. (2003). Corruption and social capital. Documento presentado en el Annual Meeting of the European Public Choice Society, Århus, April.

Beugelsdijk, S, De Groot, H.L.F. \& Van Schaik, A.B.T.M. (2002). Trust and economic growth. Documento de discusión del Tinbergen Institute, Tilburg University.

Beugelsdijk, S. \& Van Schaik, T. (2005). Social capital and growth in European regions: an empirical test. European Journal of Political Economy, 21, 301-324.

Boix, C., \& Posner, D. (1998). The origins and political consequences of social capital. British Journal of Political Science, 28, 686-693.

Bourdieu, P. (1986). The forms of capital. En J. Richardson (Ed.), Handbook of theory and research for the sociology of education (241-258). Westport, CT: Greenwood Press.

Brehm, J. \& Rahn, W. (1997). Individual-level evidence for the causes and consequences of social capital. American Journal of Political Science, 41(3), 999-1023.

Cersosimo D., Nisticò R. (2008), Social Capital in Economics. En D. Castiglione, J. van Deth \& G. Wolleb (Eds.), The Handbook of Social Capital (pp. 386-410). Oxford University Press: Oxford, pp.386-410.

Coleman, J. (1988). Social capital in the creation of human capital. American Journal of Sociology, 94, S95-S120.

Coleman, J. S. (1990). Foundations of social theory. Cambridge: Harvard University Press.

Damasio, A. (2003). El error de Descartes: la emoción, la razón y el cerebro humano. Barcelona: Crítica. 
Department of Basic Education (2011). Report on the national assessments 2011. Pretoria: Extraído el 29/12/2014 http://www.education.gov. za/LinkClick.aspx?fileticket=1U5igeVjiqg\%3D\&tabid=424\&mid=183

Delhey, J., \& Newton, K. (2003). Who trusts? The origins of social trust in seven societies. European Societies, 2, 93-137.

Dussaillant, F., \& Guzmán, E. (2014). Trust via disasters: the case of Chile's 2010 earthquake. Disasters, 38, 808-832.

EACEA (2009). National testing of pupils in Europe: objectives, organisation and use of results. Bruselas: Eurydice.

Figlio, D. \& Loeb, S. (2011). School accountability. En E. Hanushek, S. Machin \& L. Woessmann (Eds.) Handbooks in Economics, 3, 383-421. North-Holland: The Netherlands.

Frey, B. (2008). Motivation crowding theory a new approach to behavior. En Behavioral economics and public policy. Roundtable proceedings, Melbourne. Australian Government Productivity Commission.

Fukuyama, F. (1995). Trust: the social virtues and the creation of prosperity. Nueva York: Free Press.

Fukuyama, F. (1997). Social capital. The tanner lectures on human values, Brasenose College, Oxford, 12, 14 y 15 de mayo. Extraído el 29/12/2014 de http://tannerlectures.utah.edu/_documents/a-to-z/f/ Fukuyama98.pdf

Gable, A., \& Lingard, B. (2013). NAPLAN and the performance regime in Australian Schooling: A review of the policy context. Documento de investigación $N^{\circ} 4$, Social Policy Unit, University of Queensland, Brisbane.

Gambetta, D. (2000). Can we trust trust? En D. Gambetta (Ed.), Trust: Making and breaking cooperative relations (pp. 213-237). Oxford, UK: University of Oxford.

Glaeser, E. L., Laibson, D., \& Sacerdote, B. (2000). The economic approach to social capital. Documento de trabajo 7728, Cambridge, MA: National Bureau of Economic Research.

Hanifan, L.J. (1916) The rural school community center. Annals of the American Academy of Political and Social Science, 67, 130-138.

Jensen, M. C., \& Meckling,W.H. (1976). Theory of the firm: managerial behavior, agency costs and ownership structure. Journal of Financial Economics, 3(4), 305-360.

Kahneman, D., Knetsch, J. L., \& Thaler, R. H. (1986). Fairness and the assumptions of economics. Journal of Business, 59(4), S285-S300. 
Krishna, A., \& Uphoff, N. (1999). Mapping and measuring social capital: a conceptual and empirical study of collective action for conserving and developing watersheds in Rajasthan, India. Social Capital Initiative. Documento de trabajo $\mathrm{N}^{\circ}$ 13, Washington DC: Banco Mundial.

Lepper, M. R., \& Greene,D. (Eds.) (1978). Thehidden costs of reward: Newperspectives on the psychology of human motivation. Oxford: Lawrence Erlbaum.

Lowndes, V. (2000). Women and social capital: a comment on hall's social capital in Britain. British Journal of Political Science, 3(30), 533-537.

Nannicini, T., Stella, A., Tabellini, G. \& Troiano, U. (2010). Social Capital and Political Accountability, CEPR Discussion Papers 7782, C.E.P.R. Discussion Papers.

Narayan, D. \& Pritchett, L. (1999). Cents and sociability: household income and social capital in rural Tanzania. Economic Development and Cultural Change, 47, 871-897.

North, D. C. (1990). Institutions, institutional change and economic performance. Cambridge: Cambridge University Press.

Organización para la Cooperación y el Desarrollo Económicos, OECD (2011). Society at a glance 2011: OECD Social Indicators. OECD Publishing.

Organización para la Cooperación y el Desarrollo Económicos, OECD (2013). PISA 2012 Results: What makes schools successful? Resources, Policies and Practices (IV), Pisa, OECD Publishing.

Ostrom, E. (2000). Social capital, a fad or a fundamental concept? En P. Dasgupta e I. Serageldin (Eds.), Social capital, a multifaceted perspective (pp. 172-214). Washington: Banco Mundial.

Ostrom E. \& Ahn, T.K. (2009). The meaning of social capital and it's link to collective action. En G. Tinggaard Svendsen, \& E. Elgar (Eds.), Handbook of social capital: The troika of sociology, political science and economics. Chentelham: Edward Elgar.

Ostrom, E., \& Walker, J. (Eds.) (2003). Trust and reciprocity: interdisciplinary lessons from experimental research. Nueva York: Russell Sage Foundation.

Paldam, M. (2000). Social capital: one or many? Definition and measurement. Journal of Economic Surveys, 14(5), 629-53.

Putnam, R. (1993). Making democracy work. Nueva Jersey: Princeton University Press.

Putnam, R. (1995). Tuning in, tuning out: the strange disappearance of social capital in America. PS: Political Science E Politics, 4(28), 664-683. 
Putnam, R. (2000). Bowling alone: the collapse and the revival of american community. Nueva York: Simon and Schuster.

Ryan, R. M., \& Deci, E. L. (2000). Self-determination theory and the facilitation of intrinsic motivation, social development, and well-being. American Psychologist, 55, 68-78.

Sahlberg, P. (2011). Finnish Lessons: What can the world learn from educational change in Finland? Nueva York: Teachers College Press, Columbia University.

Stolle, D. (1998). Bowling together, bowling alone: the development of generalized trust in voluntary associations. Political Psychology, Special Issue: Psychological Approaches to Social Capital, 3(19), 497-525.

Nannicini, T., Stella, A, Tabellini, G., \& Troiano, U. (2010). Social capital and political accountability. American Economic Journal: Economic Policy, 2013.

Uslaner, E.M. (2001). Trust and corruption. Ponencia presentada en la Conference on Political Scandals: Past and Present, University of Salford, junio.

Vyrastekova, J. \& Garikipati, S. (2005). Beliefs and trust: an experiment. Documento de discusión CentER No 2005-88. Extraído el 29/12/2014 de http://ssrn.com/abstract=800424 o http://dx.doi.org/10.2139/ ssrn. 800424

Westlund, H., \& F. Adam (2010). Social capital and economic performance: a meta-analysis of 65 Studies. European Planning Studies, 6(18),

Woolcock, M. (1998). Social capital and economic development: toward a theoretical synthesis and policy framework. Theory and Society, 27, 151-208.

World Values Survey Wave 5 2005-2008 Official Aggregate v.20140429. World Values Survey Association, http://www.worldvaluessurvey. org/WVSDocumentationWV5.jsp Aggregate File Producer: Asep/JDS, Madrid SPAIN.

Yamagishi, T., Kikushi M., \& Kosugi, M. (1999). Trust, gullibility, and social intelligence. Asian Journal of Social Psychology, 2(1), 145-161.

Yamagishi, T. (2001). Trust as a form of social intelligence. En K. Cook (Ed.). Trust in society (pp. 121-147). Nueva York: Russell Sage Foundation.

Zak, P. \& Knack, S. (2001). Trust and growth. The Economic Journal, 111, 295-321.

Recibido: 23/12/2014

Aceptado: 29/12/2014 